(2) Open Access Full Text Article

\title{
Intranasal Delivery of miR-155-5p Antagomir Alleviates Acute Seizures Likely by Inhibiting Hippocampal Inflammation
}

This article was published in the following Dove Press journal:

Neuropsychiatric Disease and Treatment

\author{
Xu Zhou' \\ Jun Chen ${ }^{2}$ \\ Hua Tao (iD ${ }^{1,2}$ \\ Yujie Cai ${ }^{1}$ \\ Lidan Huang (D) \\ Haihong Zhou ${ }^{2}$ \\ Yanyan Chen' \\ Lili Cui ${ }^{1}$ \\ Wangtao Zhong ${ }^{2}$ \\ Keshen $\mathrm{Li}^{3}$ \\ 'Guangdong Key Laboratory of Age- \\ Related Cardiac and Cerebral Diseases, \\ Affiliated Hospital of Guangdong Medical \\ University, Zhanjiang, Guangdong \\ 52400I, People's Republic of China; \\ ${ }^{2}$ Department of Neurology, Affiliated \\ Hospital of Guangdong Medical \\ University, Zhanjiang, Guangdong \\ 52400 I, People's Republic of China; \\ ${ }^{3}$ Institute of Neurology, Affiliated \\ Hospital of Guangdong Medical \\ University, Zhanjiang, Guangdong, \\ 52400 I China; Stroke Center, Neurology \\ \& Neurosurgery Division, Clinical \\ Medicine Research Institute \& The First \\ Affiliated Hospital, Jinan University, \\ Guangzhou, Guangdong 510630, People's \\ Republic of China
}

Correspondence: Hua Tao; Keshen Li Affiliated Hospital of Guangdong Medical University No. 57, Renmindadaonan Road, Xiashan District, Zhanjiang, Guangdong 52400 I, People's Republic of China

$\mathrm{Tel} / \mathrm{Fax}+867592387$ 427;

+867592386772

Email taohua1943@126.com;

likeshen197I@I26.com
Introduction: To confront the resistance to existing antiepileptic drugs, studies have gradually begun to investigate alternative pathologies distinct from the traditional treatments that overwhelmingly target ion channels. Microglia activation is the first inflammatory response in the brain, in which miR-155-5p plays a key proinflammatory role and thus represents a promising target for inflammatory modulation in epilepsy pathologies.

Methods: In this study, a pentetrazol-induced acute seizure model was established, and the seizure degree was evaluated within $60 \mathrm{~min}$ after pentetrazol administration. Animals were then sacrificed for hippocampal tissue collection for biological experiments.

Results: Intranasal delivery of miR-155-5p antagomir (30 min before pentetrazol administration) increased the percentage of animals with no induced seizures by $20 \%$, extended the latency to generalized convulsions, and decreased seizure severity. In addition, miR-155-5p antagomir treatment alleviated hippocampal damage and decreased the expression of typical inflammatory modulators (TNF- $\alpha$, IL-1 $\beta$ and IL-6). Further research revealed that intranasal delivery of miR-155-5p antagomir significantly decreased the relative level of miR-155-5p and increased the expression of its targets $\operatorname{LXR} \alpha$ and SOCS1 in IBA1-labeled microglial cells in the hippocampus.

Conclusion: These findings demonstrate that intranasal delivery of miR-155-5p antagomir alleviated acute seizures, likely by blocking hippocampal inflammation. However, other potential mechanisms of the effects of miR-155-5p antagomir and its long-term safety for epilepsy treatment remain to be investigated.

Keywords: intranasal delivery, epilepsy, pentetrazol, inflammatory response, miR-155-5p

\section{Introduction}

Epilepsy, characterized by recurrent seizures, is one of the most common brain disorders, affecting almost 50 million people worldwide. Despite pharmacological progress in regulating various ion channels, such as oxcarbazepine for sodium channels and topiramate for multiple ion channels, epilepsy is rarely cured but rather can be temporarily controlled. ${ }^{1}$ Even so, nearly one-third of epilepsy patients still suffer from repeated convulsions due to resistance to the existing drugs. To overcome this dilemma, researchers have gradually begun to focus on other pathologies distinct from the traditional changes in ion channels. Among these, the role of neuroinflammation in epilepsy has attracted increasing attention in recent years. ${ }^{2}$

Currently, several lines of evidence support the involvement of inflammation in epileptogenesis. First, a series of clinical studies observed epileptic seizures in classical 
inflammatory diseases such as systemic lupus, vasculitis and multiple sclerosis, ${ }^{3}$ and in patients with such diseases, antiinflammatory therapies are usually more effective than standard antiepilepsy drug therapies at disease onset. ${ }^{4}$ Second, lipopolysaccharide is a proinflammatory agent that is able to induce both systemic and brain inflammation, potentially resulting in brain excitability and epileptic seizures in rodents. ${ }^{5}$ Meanwhile, interleukin-1 $\beta$ (IL-1 $\beta$ ), tumor necrosis factor- $\alpha(\mathrm{TNF}-\alpha)$ and interleukin- 6 (IL-6) are considered classical proinflammatory mediators. Interestingly, IL-1 $\beta$ exacerbates seizures in animal models by enhancing susceptibility to seizure-inducing stimuli, ${ }^{6,7}$ and spontaneous seizures take place in transgenic mice overexpressing TNF- $\alpha$ and IL- $6 .{ }^{8-11}$ Indeed, inflammatory mediators, activated astrocytes and microglia, and other indicators of inflammation have been found in the resected hippocampus of patients with temporal lobe epilepsy (TLE), the most common type of refractory epilepsy. ${ }^{2}$ This evidence suggests that inflammatory injuries in the brain predispose patients to the occurrence of epileptic activities.

On the other hand, neuroinflammation could be rapidly induced in rodents after chemically and electrically induced seizures. ${ }^{12}$ Subsequent cascades of inflammation are usually long lasting, along with the occurrence of spontaneous seizures. For example, IL- $1 \beta$ expression in glial cells remains elevated for up to 60 days after experimental epileptic seizures, ${ }^{13}$ and spontaneous seizures occur in the chronic stage of the lithium-pilocarpineinduced TLE model. ${ }^{14}$ To some extent, epileptic seizures are capable of inducing neuroinflammation, which then enhances epileptic activities; thus, blocking this vicious circle between seizure and neuroinflammation should be a promising strategy for treating epilepsy. In fact, previous studies have reported that antiinflammatory steroids displayed anticonvulsant effects in some patients with drugresistant epilepsy. ${ }^{15,16}$ However, steroids interfere with the immune system, often followed by a series of side effects. ${ }^{17}$ In addition, neuroinflammation is extremely complex and usually varies under different seizureinducing stimuli, which act on various cell types and involve multiple inflammatory cytokines. ${ }^{2}$ Hence, determining whether other inflammatory mediators could be used to target neuroinflammation for the treatment of epilepsy is an urgent matter.

MicroRNAs (miRNAs) are small molecules 22 nucleotides long that regulate biological activities by suppressing the translation of their target genes; thus, miRNAs can be easily delivered intranasally to treat brain diseases due to their ability to bypass the blood-brain barrier (BBB). ${ }^{18,19}$ Indeed, intranasal delivery of miRNA agomir or antagomir has been developed as a practical and efficient strategy to treat brain disorders. ${ }^{20}$ Numerous studies have shown a key role of miRNAs, especially miR-146a-5p and miR-155-5p, in the regulation of inflammatory processes. Our team found that intranasal delivery of miR-146a-5p agomir improved epileptic seizures and hippocampus inflammation. ${ }^{21}$ In comparison, miR-155-5p plays a proinflammatory role in microglia by inhibiting the expression of suppressor of cytokine signaling 1 (SOCS1) and liver X receptor $\alpha(\mathrm{LXR} \alpha)^{22,23}$ Moreover, the expression of miR-155-5p is increased in the hippocampus of an epilepsy animal model and patients with mesial TLE. ${ }^{24}$ Hence, intranasal delivery of miR-155-5p antagomir appears to be a promising way to control hippocampal inflammation in epilepsy pathologies.

To preliminarily confirm the hypothesis that intranasal delivery of miR-155-5p antagomir alleviates acute seizures by countering hippocampal inflammation, we established a pentetrazol-induced model in the present study and examined the protective effects of artificially synthesized miR-155$5 \mathrm{p}$ antagomir on acute seizures and inflammatory injuries in the hippocampus, expecting to supply a novel strategy for the translational development of epilepsy therapies.

\section{Materials and Methods}

\section{Experimental Animals}

A total of 108 male Sprague Dawley (SD) rats were obtained from the Animal Center of Guangdong Medical University, Zhanjiang, China. The rats were bred under a humidity of $55 \%$ to $65 \%$ and a temperature of $22^{\circ} \mathrm{C}$ to $26^{\circ} \mathrm{C}$. A light-dark cycle was implemented in accordance with circadian rhythm. After a one-week adaptation to the environment along with free access to food and water, rats (body weight, $248 \pm 30 \mathrm{~g}$ ) were used in the following experiments. The study was performed in accordance with the Guide for the Care and Use of Laboratory Animals (Ministry of Science and Technology of China, 2006) and was approved by the Animal Ethics Committee of Guangdong Medical University.

\section{Dynamic Changes in miR-155-5p After Intranasal Delivery of miR-155-5p \\ Antagomir}

Following the manufacturer's instructions, $200 \mathrm{nmol}$ of miR-155-5p antagomir (sequence information: 5'-cholacccctatcacgattagcattaa-3'; Genesky Biotech, Shanghai, China) was dissolved in $2 \mathrm{~mL}$ RNase-free water, forming 
the miR-155-5p antagomir solution (100 nmol/mL) for intranasal administration in the subsequent experiments. Referring to a previous study, ${ }^{25}$ the rats were fixed in a simple mouse holder (Yuyan Instruments, Shanghai, China) and placed in a supine position; the miR-155-5p antagomir solution was then administered via a $10-\mu \mathrm{L}$ pipette at a rate of $2 \mu \mathrm{L}$ per drop and 12 drops per minute, alternating between each naris every 2 drops. According to the study protocol, a total of 18 rats were randomly selected and equally divided into three groups to receive $\mathrm{miR}-155-5 \mathrm{p}$ antagomir $(10 \mathrm{nmol} / \mathrm{kg}$ body weight). The three groups were sacrificed for the collection of the hippocampus and cortex at $30 \mathrm{~min}, 60 \mathrm{~min}$ and $120 \mathrm{~min}$ after intranasal delivery of miR-155-5p antagomir. Another group of 6 rats received equal-volume RNasefree water $(0.1 \mathrm{~mL} / \mathrm{kg}$ body weight $)$ as the vehicle control and were immediately sacrificed after intranasal administration $(0 \mathrm{~min})$. To identify the effectiveness of intranasal miR-155-5p antagomir delivery in the hippocampus and cortex, we quantitated the relative miR-155-5p levels in the hippocampus and cortex using real-time quantitative polymerase chain reaction (qPCR) and further analyzed their dynamic changes after intranasal miR-155-5p antagomir delivery by comparing miR-155-5p expression with that in the vehicle control.

\section{Relative miR-155-5p Levels in Two Acute Seizure Models}

In total, 23 rats were randomly selected to establish two acute seizure models. Generalized convulsions were successfully induced in 6 rats using the pilocarpine-induced model and another 6 rats using the pentetrazol-induced model. The protocol for the pilocarpine-induced model was as follows: Lithium chloride $(125 \mathrm{mg} / \mathrm{kg}$ body weight, i.p.; Amresco LLC, Solon, China) was used to enhance the sensitivity of pilocarpine, and scopolamine $(1 \mathrm{mg} / \mathrm{kg}$ body weight, i.p.; Tomax, Shenzhen, China) was injected $20 \mathrm{~h}$ later to limit cholinergic effects and the risk of unexpected death, followed by pilocarpine administration (50 mg/kg body weight, i.p.; Cayman, Ann Arbor, USA) $30 \mathrm{~min}$ later. The degree of seizures was evaluated according to the Racine scale, and if seizures did not emerge or reach level 4, administration of pilocarpine $(10 \mathrm{mg} / \mathrm{kg}$ body weight) was repeated every $10 \mathrm{~min}$ until a total dose of pilocarpine of $100 \mathrm{mg} / \mathrm{kg}$ body weight. For the pentetrazol-induced model, pentetrazol $(60 \mathrm{mg} / \mathrm{kg}$ body weight, i.p.; Cayman, Ann Arbor, USA) was administered, and if seizures did not emerge or reach level 4, administration of pentetrazol (20 $\mathrm{mg} / \mathrm{kg}$ body weight) was repeated every $10 \mathrm{~min}$ until a total dose of pentetrazol of $120 \mathrm{mg} / \mathrm{kg}$ body weight. In addition, another group of 6 rats was randomly selected as a control group. Finally, for the identification of the relative miR-155-5p levels in the two acute seizure models, the 18 rats were sacrificed for the collection of the hippocampus.

\section{Electrical Activities of the Brain in the Pentetrazol-Induced Model}

A tethered electroencephalogram/electromyography (EEG/ EMG) acquisition system (Pinnacle Technologies Inc., Lawrence, USA) was applied to determine if epileptiform discharges occurred in the pentetrazol-induced epilepsy model in the present study. Here, 1 rat was randomly selected and anesthetized using 3\% chloral hydrate (10 $\mathrm{mL} / \mathrm{kg}$ body weight, intraperitoneally (i.p.), SigmaAldrich, St. Louis, USA). Upon loss of awareness, four pilot holes were drilled through the skull for the implantation of intracranial electrodes, and then a preamplifier head mount was affixed to the skull using dental acrylic, as described in a previous study. ${ }^{26}$ After a one-week recovery period with free access to food and water, no abnormalities, such as infection, hemorrhage or seizure, were observed, and then acute seizures and related brain electrical activities within 60 min after pentetrazol administration were recorded. In addition, brain electrical activities before pentetrazol administration were recorded for selfpaired analysis.

\section{Grouping and Behavior Observations}

A total of $60 \mathrm{SD}$ rats were randomly selected and divided into three groups: The seizure model group $(n=20)$, which was used for the induction of the pentetrazol model, the antagomir treatment group $(n=20)$, and the control group $(n=20)$. In comparison with the seizure model group, the antagomir treatment group received an extra administration of miR-155-5p antagomir $(100 \mathrm{nmol} / \mathrm{mL}, 10 \mathrm{nmol} / \mathrm{kg}$ body weight) intranasally $30 \mathrm{~min}$ before pentetrazol administration. Within $60 \mathrm{~min}$ after pentetrazol administration, the percentage of rats with no induced seizures, the latency to generalized convulsions, and the seizure severity were recorded to identify the effects of miR-155-5p antagomir on epileptic seizures. In the case of generalized convulsions lasting more than $30 \mathrm{~min}, 3 \%$ chloral hydrate (2 $\mathrm{mL} / \mathrm{kg}$ body weight, i.p.; Sigma-Aldrich, St. Louis, 
USA) was administered every 5 min until seizure cessation to reduce unexpected deaths of the experimental rats before their prespecified time of sacrifice. According to the severity of rats with epileptic seizures, the manifestations of the Racine scale were classified into 5 levels: (1) Twitching of facial muscles; (2) Nodding of the head; (3) Lifting or clonus of unilateral forelimb; (4) Bilateral forelimb clonus and rearing; (5) loss of posture exhibited by falling or twisting. Referring to previous standards, ${ }^{27,28}$ level 4 was defined as the threshold for generalized convulsions in this study. After $60 \mathrm{~min}$ of pentetrazol administration, 9 rats, 9 rats and 2 rats per group were randomly used for miRNA/mRNA quantitation (real-time qPCR), protein quantitation (Western blot, WB; enzyme-linked immunosorbent assay, ELISA) and hippocampus staining (hematoxylin-eosin staining, HE; Nissl staining; terminal deoxynucleotidyl transferase-mediated dUTP-biotin nick end labeling assay, TUNEL; double immunofluorescence labeling), respectively.

\section{Molecular Biology Experiments}

To identify molecular changes in the brain, rats were sacrificed by decapitation under deep anesthesia using 10\% chloral hydrate (3 $\mathrm{mL} / \mathrm{kg}$ body weight, i.p.; Sigma-Aldrich, St. Louis, USA), and brain tissues were quickly isolated for the following experiments: (1) Real-time qPCR: Total RNA was extracted under an RNase-free condition via an RNA extraction kit (Thermo Fisher Scientific, Waltham, USA) and then used for reverse transcription via a First Strand cDNA Synthesis Kit (Thermo Fisher Scientific, Waltham, USA) in line with the manufacturer's instructions. The cDNA products were further amplified by a Light-Cycler 480 sequence detector system (Roche Applied Science, Penzberg, Germany), and the primers were as follows: miR-155, 5'-cagcctacacg gtgggagc-3' (F), 5'-ctgctctgagtcattgtgctgg-3' (R), LXR $\alpha, 5^{\prime}$-ga gtcatccgagcctacagc-3'(F), and 5'-gaaggagcgcctgttacact-3'(R), SOCS1, 5'-catcctcgtcctcgtcttcg-3'(F), and 5'-ttaagagggatgcg tgccag-3'(R), and GAPDH, 5'-gccagcctcgtctcatagac-3'(F), and $5^{\prime}$-agtgatggcatggactgtgg- $3^{\prime}(\mathrm{R})$. Finally, the relative levels of miR-155-5p and its targets LXR $\alpha$ and SOCS1 mRNAs were calculated using the $2^{-\triangle \Delta C T}$ method, and U6 and GAPDH were used as internal references for miR-155-5p and LXR $\alpha$ and SOCS1 mRNAs, respectively. (2) WB: Protein extracted from brain tissues was transferred to polyvinylidene fluoride membranes (Millipore, Darmstadt, Germany) for $90 \mathrm{~min}$ at $400 \mathrm{~mA}$. After the membranes were incubated with the primary rabbit polyclonal anti-LXR $\alpha$ antibody (diluted 1:100; Santa Cruz, Texas, USA), mouse monoclonal anti-SOCS1 antibody (diluted 1:100; Santa Cruz, Texas, USA) or primary mouse monoclonal anti- $\beta$ actin antibody (diluted 1:100; Santa Cruz, Texas, USA) used as an internal control, they were reacted with goat anti-rabbit secondary antibody (diluted 1:100; Beyotime, Beijing, China) or goat anti-mouse secondary antibody (diluted 1:100; Beyotime, Beijing, China) at $37^{\circ} \mathrm{C}$ for $1 \mathrm{~h}$. The immunoreactive bands were detected using an enhanced chemiluminescence substrate kit (Beyotime, Shanghai, China). (3) ELISA: The concentrations of IL- $1 \beta$, TNF- $\alpha$ and IL- 6 in the hippocampus were detected via ELISA kits (R\&D Systems, Minneapolis, USA) in line with the manufacturer's instructions, and their absorbance was detected using an ELISA reader (Bio-Rad Laboratories, Hercules, USA).

In addition, hippocampal staining was performed as follows: (1) HE, Nissl and TUNEL staining: The hippocampi were fixed in $4 \%$ paraformaldehyde overnight, dehydrated in a graded ethanol series, embedded in paraffin and then cut into 4- $\mu \mathrm{m}$-thick slices, which were deparaffinized and then used for HE and Nissl staining for examination of histopathological injuries under light microscopy (Zeiss Axiovert S100TV, Jena, Germany); TUNEL staining was used to determine in situ apoptosis via a TUNEL Apoptosis Detection Kit (Roche Applied Science, Penzberg, Germany) according to the manufacturer's instructions. (2) Double immunofluorescence labeling: The hippocampi were fixed and then cut into $4-\mu \mathrm{m}$-thick slices, which were incubated with the primary antibodies primary rabbit polyclonal anti-LXR $\alpha$ antibody (diluted 1: 100; Santa Cruz, Texas, USA), mouse monoclonal anti-SOCS1 antibody (diluted 1: 100; Santa Cruz, Texas, USA), and mouse monoclonal anti-ionized calcium binding adapter molecule 1 (IBA1) (diluted 1: 100; Santa Cruz, Texas, USA); these slices were further treated with 40,6-diamidino-2-phenylindole dihydrochloride (Sigma, St. Louis, USA) to identify nuclei. Double immunofluorescent-labeled slices were detected by laser scanning confocal microscopy (Leica Microsystems, Wetzlar, Germany) and an Olympus IX 70 inverted microscope (Olympus America, NY, USA).

\section{Statistical Analysis}

Measurement data are displayed as the mean \pm standard error of the mean and compared using a two independent samples $t$-test for two groups and the ANOVA with a post hoc test for three groups or more. Statistical analyses were performed with SPSS 19.0 (IBM, New York, USA), and a two-tailed $\mathrm{p} \leq 0.05$ was considered statistically significant. Graph Pad 
Prism 5 (GraphPad, New York, USA) was applied to illustrate the findings. In addition, all staining images were analyzed by researchers who were blinded to the experimental groups.

\section{Results}

\section{Effectiveness of miR-155-5p Antagomir via Intranasal Delivery and the \\ Pentetrazol-Induced Acute Seizure Model}

As shown in Figure 1A, the relative miR-155-5p level in the hippocampus was reduced to 0.61 -fold at $30 \mathrm{~min}$ after intranasal delivery of miR-155-5p antagomir and further reached a 0.59 and 0.54 -fold decrease at $60 \mathrm{~min}$ and $120 \mathrm{~min}$, respectively, in comparison with the levels in the vehicle control sacrificed at $0 \mathrm{~min}$. Similarly, the relative miR-155-5p levels in the cortex of the miR-155-5p antagomir-treated groups were reduced to 0.76 -fold at $30 \mathrm{~min}$ and
0.73 -fold at both $60 \mathrm{~min}$ and $120 \mathrm{~min}$ compared with the levels in that of the vehicle control group sacrificed at $0 \mathrm{~min}$. Via statistical analysis, compared with the miR-155-5p levels in the vehicle control sacrificed at $0 \mathrm{~min}$, the levels were significantly lower in the hippocampus after intranasal delivery of miR-155-5p antagomir, indicating that miR155-5p antagomir could function in the hippocampus via intranasal delivery. In comparison with the cortex, the hippocampus is a key region of inflammatory injuries for epilepsy pathologies and expressed a lower level of miR$155-5 \mathrm{p}$ at $60 \mathrm{~min}$ after delivery that was maintained for 60 $\min$. As to the difference between hippocampus and cortex, it might result from the condition that intranasal delivery bypasses the blood-brain barrier via olfactory- and trigeminal-associated extracellular pathways, ${ }^{29,30}$ and these pathways are relatively adjacent to hippocampus instead of cortex from the perspective of regional anatomy.

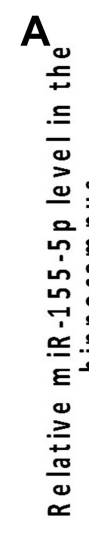

B

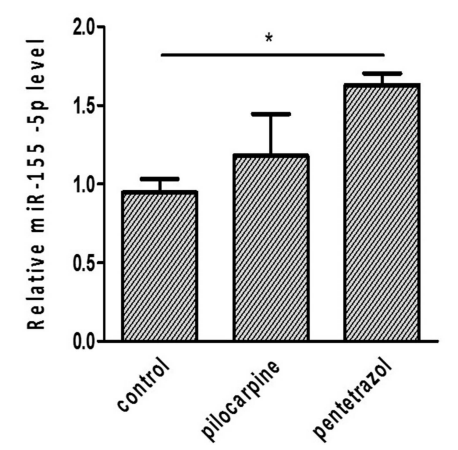

Normal state before injection of pentetrazol

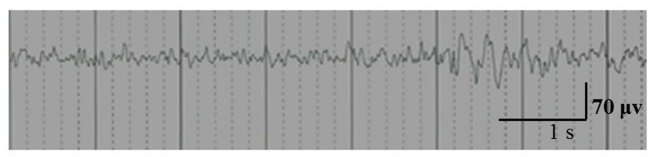

Epileptic discharges induced by pentetrazol

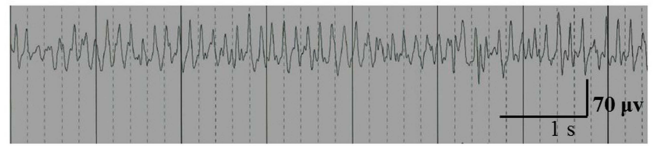

Figure I The effectiveness of miR-I55-5p antagomir intranasal delivery and the acute epilepsy model induced by pentetrazol. (A) Dynamic changes in relative miR-I55-5p levels after intranasal delivery of miR-155-5p antagomir. Based on real-time $q P C R$, the relative miR- $155-5 p$ levels were $1.00 \pm 0.10,0.61 \pm 0.10,0.56 \pm 0.09$ and $0.54 \pm 0.10$ in the rats sacrificed at $0 \mathrm{~min}(n=6), 30 \mathrm{~min}(n=6), 60 \mathrm{~min}(n=6)$ and $120 \mathrm{~min}(n=6)$ in the hippocampus, respectively; the relative miR-155-5p levels were $1.00 \pm 0.05,0.76 \pm 0.14,0.73$ \pm 0.08 and $0.73 \pm 0.09$ in the rats sacrificed at $0 \mathrm{~min}(n=6), 30 \mathrm{~min}(n=6), 60 \mathrm{~min}(n=6)$ and $120 \mathrm{~min}(n=6)$ in the cortex, respectively. (B) Relative miR-I55-5p levels in two acute seizure models. Based on real-time qPCR, the relative miR-155-5p levels were $0.95 \pm 0.09,1.18 \pm 0.27$ and $1.63 \pm 0.08$ in the control ( $n=6$ ), the pilocarpine-induced model ( $n=6$ ) and the pentetrazol-induced model $(n=6)$, respectively. (C) Epileptic discharges of the pentetrazol-induced acute seizure model. Compared with the normal state, the state after pentetrazol administration was characterized by epileptic discharges (sharp wave, 6-7 times per second) along with generalized convulsions using the EEG/EMG acquisition system. ${ }^{*} \mathrm{p}<0.05$ and ${ }^{* *} \mathrm{p}<0.01$. 
As shown in Figure 1B, via statistical analysis, the relative miR-155-5p levels increased to 1.42 and 1.59 -fold in the pilocarpine- and pentetrazol-induced models, in comparison with the levels in the control group, but the latter model seemed to be a better model for this study due to higher fold change along with smaller magnitude with an increasing trend of miR-155-5p levels, indicating that the model is fit for exploring the effects of miR-155-5p antagomir on epileptic seizures. In addition, as shown in Figure 1C, epileptic discharges along with generalized convulsions were observed after pentetrazol administration, confirming the effectiveness of the pentetrazol protocol used in the subsequent experiments of this study.

\section{Behavioral Observations After Intranasal Delivery of miR-155-5p Antagomir in the Pentetrazol-Induced Acute Seizure Model}

As shown in Figure 2, the percentage of rats without seizures increased by $20 \%$ (A), the percentage of rats (the Racine level $\geq 4$, defined as generalized convulsions) decreased by $20 \%$ (B), and the latency to generalized convulsion after pentetrazol administration was significantly extended $(\mathrm{C}$ and $\mathrm{D})$ in the antagomir treatment group compared with those in the seizure model group. All these findings indicate that intranasal delivery of miR-155-5p antagomir improved the incidence and severity of acute seizures in the pentetrazol-induced model.
A

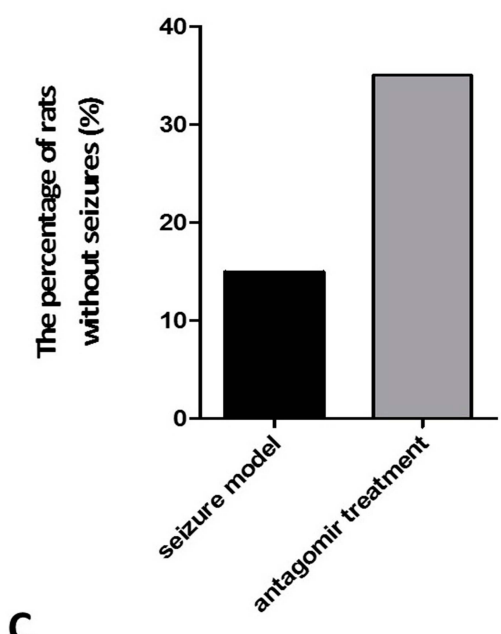

C

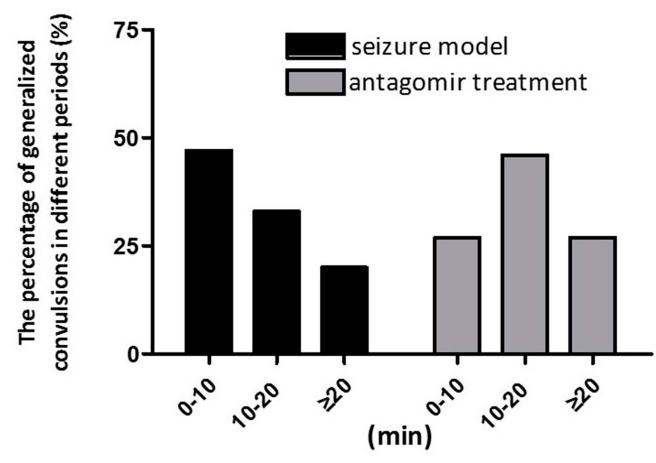

B

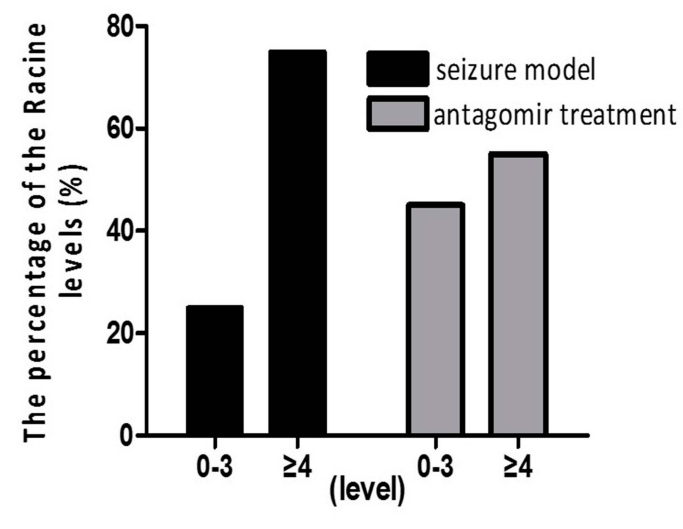

D

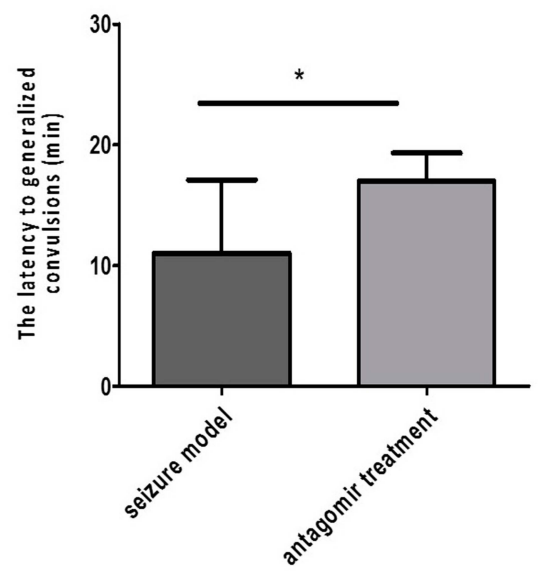

Figure 2 Behavioral observations within 60 min after pentetrazol administration in the pentetrazol-induced model. (A) The percentages of rats without seizures were $15 \%$ $(3 / 20)$ and $35 \%(7 / 20)$ in the seizure model and antagomir treatment groups, respectively. (B) The percentage of rats with a Racine level $\geq 4$ (defined as generalized convulsions) were $75 \%(15 / 20)$ and $55 \%(11 / 20)$ in the seizure model and antagomir treatment groups, respectively. (C) The percentages of generalized convulsions were $47 \%(7 / / 5)$ and $21 \%(3 / 1 \mathrm{I})$ at $0-10 \mathrm{~min}, 33 \%(5 / 15)$ and $55 \%(6 / 1 \mathrm{I})$ at $10-20 \mathrm{~min}$, and $20 \%(3 / 15)$ and $27 \%(3 / \mathrm{II})$ at more than $20 \mathrm{~min}$ after pentetrazol administration in the seizure model and antagomir treatment groups, respectively. (D) The latencies to generalized convulsions were $11.0 \pm 6.1 \mathrm{~min}$ and $17.0 \pm 7.7 \mathrm{~min}$ in the seizure model $(\mathrm{n}=15$ ) and antagomir treatment groups $(n=11)$, respectively. ${ }^{*} p<0.05$. 


\section{Expression of miR-155-5p and Its Targets $\mathrm{LXR} \alpha$ and SOCSI After Intranasal Delivery of miR-I55-5p Antagomir in the Pentetrazol-Induced Model}

As shown in Figure 3, the relative level of miR-155-5p in the hippocampus was significantly lower in the antagomir treatment group than in the seizure model group (A), and the hippocampal expression of $\operatorname{LXR} \alpha$ mRNA and SOCS1 mRNA was increased with mild significant $(p=0.026)$ for the former and even none $(p=0.517)$ for the latter (B). Furthermore, increasing trend of LXR $\alpha$ and SOCS1 protein in the hippocampus of the antagomir treatment group was observed compared with the seizure model group (C). Intriguingly, the expression of $\operatorname{LXR} \alpha$ and SOCS1 protein in IBA1labeled microglia cells was higher in the antagomir treatment group than in the seizure model group (Figures 4 and 5), which supports the hypothesis that intranasal delivery of miR-155-5p antagomir downregulated miR-155-5p expression, thereby resulting in a higher expression of LXR $\alpha$ and SOCS1 in hippocampal microglia.
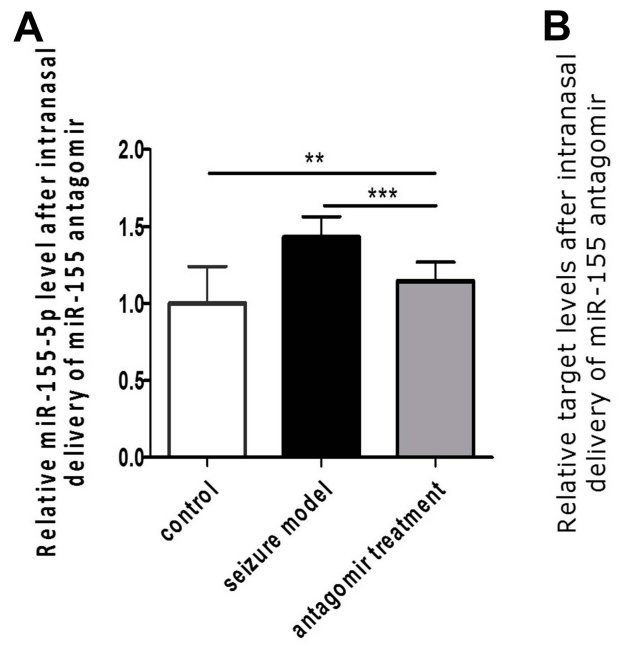

C
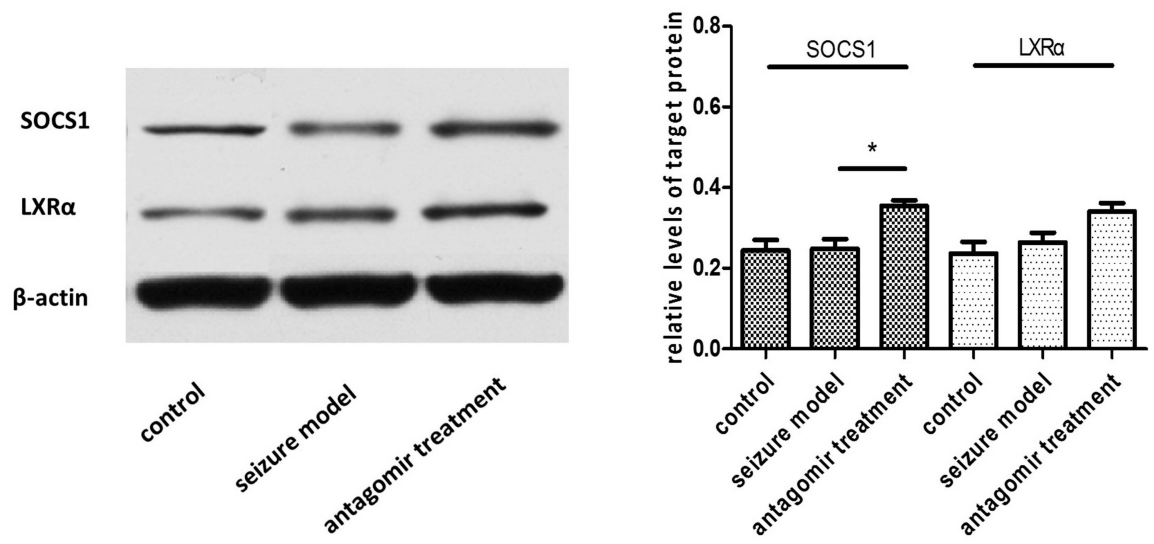

Figure 3 Expression of miR-155-5p and its targets LXR $\alpha$ and SOCSI at 60 min after pentetrazol administration in the pentetrazol-induced acute epilepsy model. (A) Based on real-time $q P C R$, the relative miR- $155-5 p$ levels in the hippocampus were $1.00 \pm 0.08,1.43 \pm 0.13$ and $1.15 \pm 0.12$ in the control group ( $n=9$ ), the seizure model group ( $n=9$ ) and the antagomir treatment group $(n=9)$, respectively; via statistical analysis, the relative level of miR- $155-5 p$ in the hippocampus of the antagomir treatment group decreased to $34 \%$ of that in the seizure model group. (B) Based on real-time qPCR, the relative LXR levels in the hippocampus were I.00 $\pm 0.03, I .03 \pm 0.07$ and I.59 \pm 0.07 in the control group $(n=9)$, the seizure model group $(n=9)$ and the antagomir treatment group $(n=9)$, respectively; the relative $L X R \alpha$ levels in the hippocampus were 1 . $10 \pm 0.04$, $1.22 \pm 0.02$ and $1.4 I \pm 0.04$ in the control group $(n=9)$, the seizure model group $(n=9)$ and the antagomir treatment group ( $n=9)$, respectively; via statistical analysis, the expression of LXR $\alpha$ and SOCSI mRNA in the hippocampus of the antagomir treatment group increased to 1.6 and 1.4 times that in the hippocampus of the seizure model group. (C) WB indicated increasing trend of $L X R \alpha$ and SOCSI protein in the hippocampus of the antagomir treatment group ( $n=3$ ) compared with the seizure model group $(n=3) . * p<0.05, * * p<0.01$ and $* * * p<0.001$. 

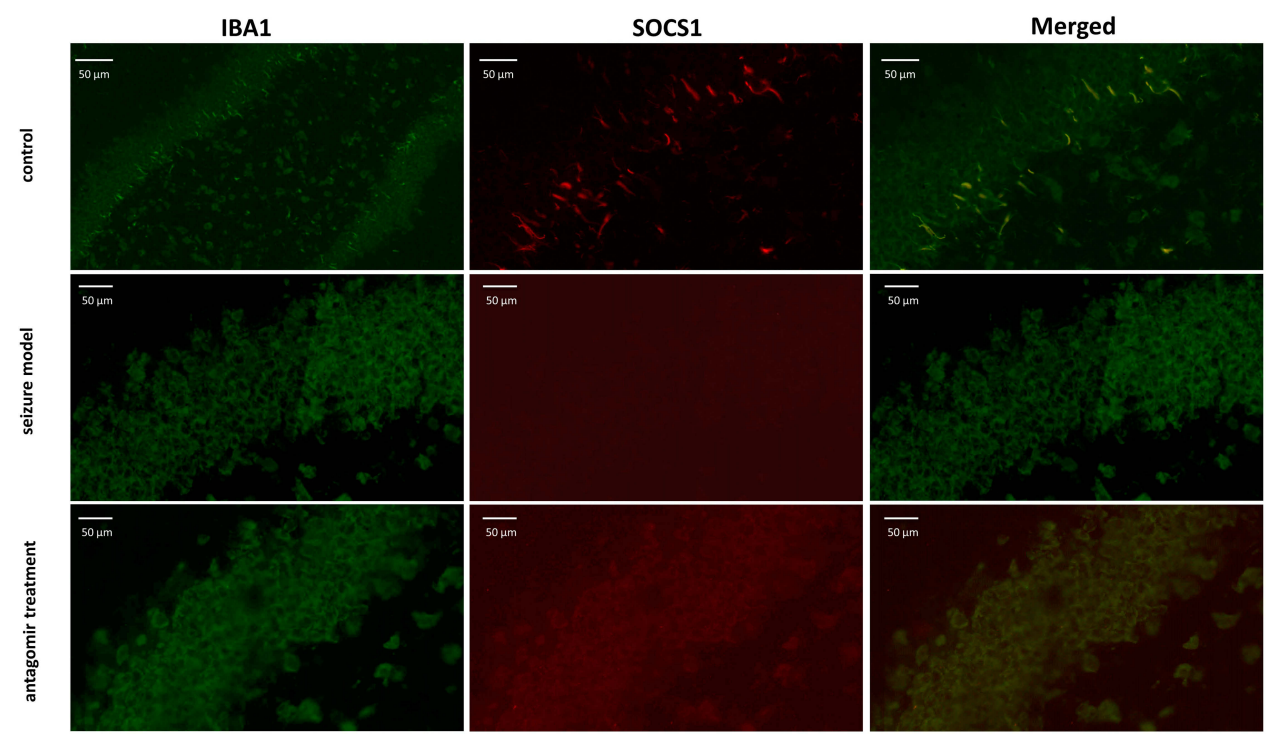

Figure 4 Double immunofluorescence labeling showed $L X R \alpha$ protein (red) in IBAI-labeled microglial cells (green) of hippocampal tissues at 60 min after pentetrazol administration in the pentetrazol-induced acute seizure model. In the control group, IBAI-labeled microglial cells were relatively small and presented as a short-bar shape, indicating that they were in a resting state, and $L X R \alpha$ could be seen clearly within them. In the seizure model group, labeled microglial cells became spherical in shape and overlapped with each other, indicating that they were activated, but the expression of LXR $\alpha$ was not significantly upregulated. In the antagomir treatment group, labeled microglial cells still appeared spherical in shape and overlapped with each other, indicating that they were in an activated state, and the expression of LXR $\alpha$ was significantly upregulated compared with the seizure model group.

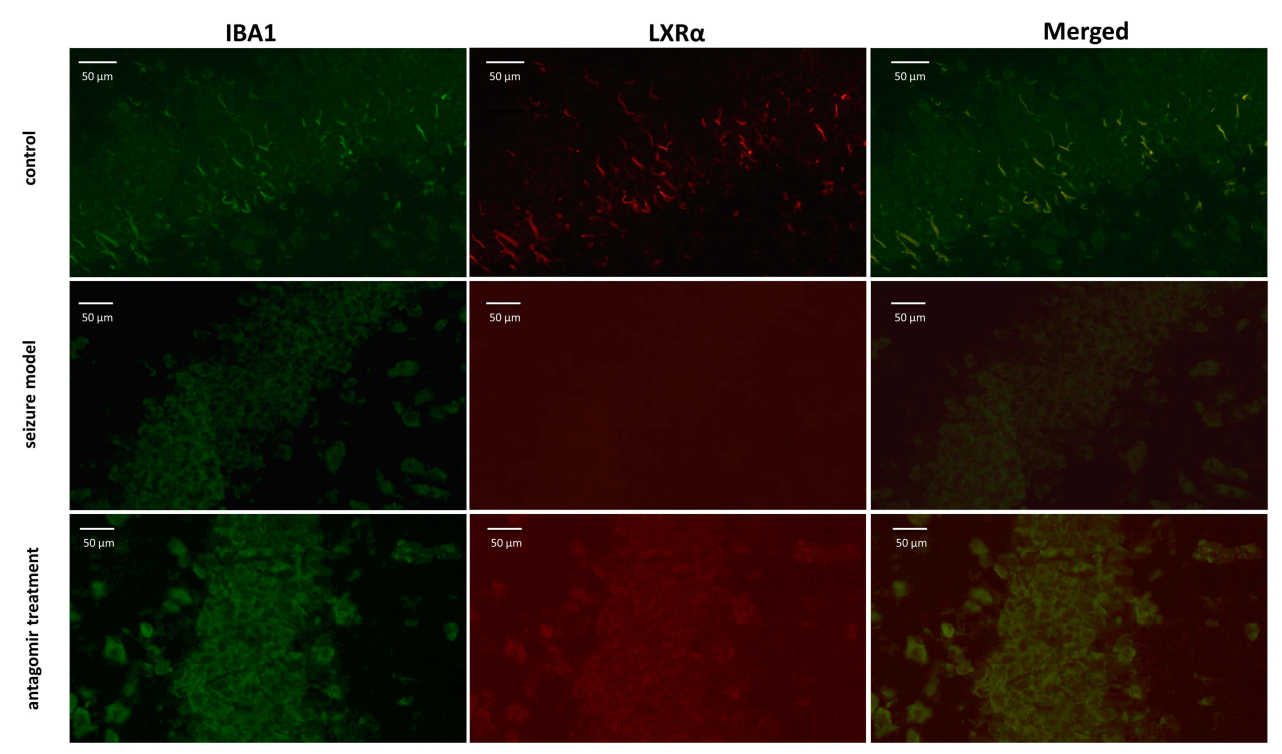

Figure 5 Double immunofluorescence labeling showed LXR $\alpha$ protein (red) in IBAI-labeled microglia (green) in the hippocampus at 60min after the injection of pentetrazol in the acute seizure model. In the control group, IBAI-labeled microglia were small with a short-bar shape, supporting that these microglia were in a resting state, and LXR $\alpha$ could be observed clearly within them. In the seizure model group, labeled microglia were spherical instead of a short-bar shape, indicating that they were activated, but LXR $\alpha$ expression did not significantly increased. In the antagomir treatment group, labeled microglia were still spherical, but the immunofluorescent signal of $L X R \alpha$ expression significantly increased compared with the seizure model group.

\section{Expression of Typical Inflammatory \\ Modulators After Intranasal Delivery of miR-I55-5p Antagomir in the Pentetrazol-Induced Model}

As shown in Figure 6, the expression of IL-1 $\beta$, TNF- $\alpha$ and IL-6 in the hippocampus of the antagomir treatment group was reduced to $79.7 \%, 74.7 \%$ and $70.0 \%$ of that in the seizure model group, respectively. However, the expression levels in the antagomir treatment group were still higher than the levels in the control group. These results indicate that intranasal delivery of miR-155-5p antagomir inhibited the inflammatory response in the pentetrazolinduced epilepsy model but did not completely block it. 
A

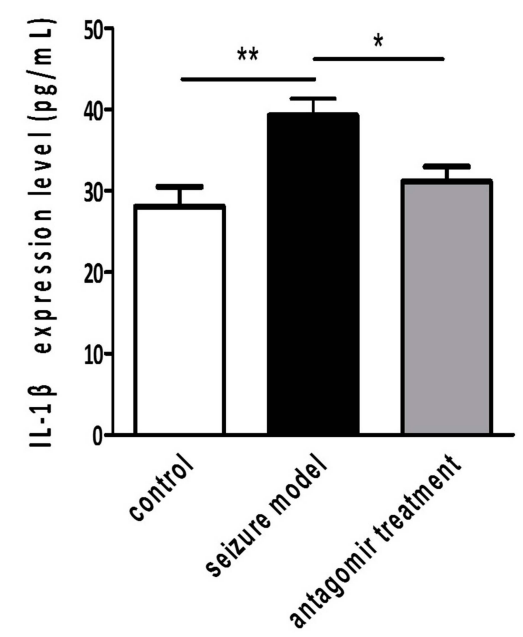

B

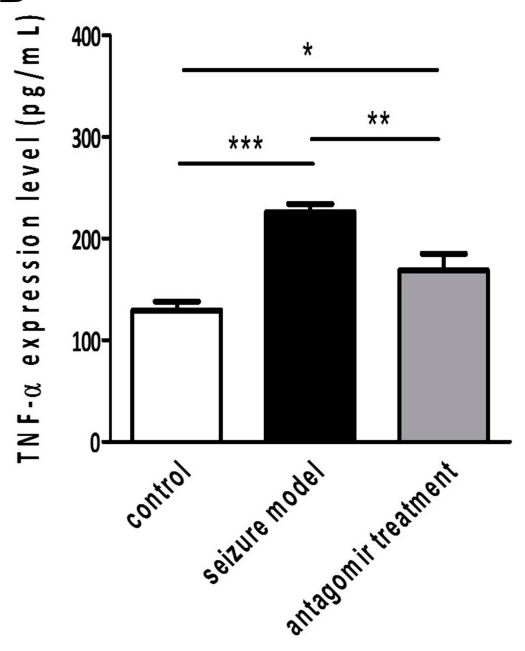

C

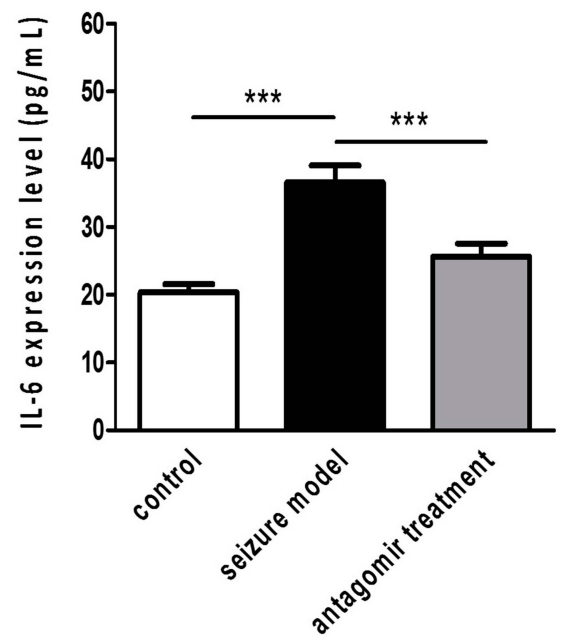

Figure 6 Expression of typical inflammatory modulators at 60 min after pentetrazol administration in the pentetrazol-induced acute seizure model. Based on ELISA, the concentrations of IL-I $\beta$ (A), TNF- $\alpha$ (B) and IL-6 (C) were $28.1 \pm 2.5 \mathrm{pg} / \mathrm{ml}, 39.3 \pm 2.1 \mathrm{pg} / \mathrm{ml}$ and $31.1 \pm 1.9 \mathrm{pg} / \mathrm{ml}$ in the hippocampus of the control group ( $\mathrm{n}=9$ ), $129.5 \pm 8.8 \mathrm{pg} /$ $\mathrm{ml}, 226.2 \pm 8.0 \mathrm{pg} / \mathrm{ml}$ and $169.0 \pm 16.2 \mathrm{pg} / \mathrm{ml}$ in the hippocampus of the seizure model group $(\mathrm{n}=9)$, and $20.4 \pm 1.2 \mathrm{pg} / \mathrm{ml}, 36.6 \pm 7.5 \mathrm{pg} / \mathrm{ml}$ and $25.6 \pm 2.0 \mathrm{pg} / \mathrm{ml}$ in the hippocampus of the antagomir treatment group $(n=9)$, respectively. ${ }^{*} p<0.05,{ }^{* *} p<0.01$ and ${ }^{* * *} p<0.001$.

\section{Protective Effects of miR-I55-5p}

\section{Antagomir on the Hippocampus in the} Pentetrazol-Induced Acute Seizure Model

As shown in Figure 7, compared with the hippocampal tissue of the control group, the hippocampal tissue of the seizure model group underwent severe structural breakdown (shown by HE staining) and displayed fewer Nissl bodies. However, intranasal delivery of miR-155-5p antagomir significantly alleviated hippocampal damage in the antagomir treatment group. In addition, there was no significant apoptosis of hippocampal tissues (shown by TUNEL and DAPI staining) in the control, seizure model or antagomir treatment groups. Regarding apoptosis, this timepoint is likely too early to observe apoptotic cell death with a TUNEL assay. Our results cannot exclude the possibility that apoptosis of hippocampal tissues may emerge later after $1 \mathrm{~h}$ post-pentetrazol treatment or even in the latent and chronic stages. Nonetheless, our results suggest that apoptosis is not significantly involved in protective effects of miR-155-5p antagomir on acute seizures. Combined with the hippocampal damage shown by HE and Nissl staining, these findings indicate that miR-155-5p antagomir plays a protective role in the pentetrazol-induced acute seizure model by inhibiting hippocampal damage but not apoptosis.

\section{Discussion}

This study first observed that intranasal delivery of miR-155$5 \mathrm{p}$ antagomir alleviated acute seizures and hippocampal inflammation in the pentetrazol-induced epilepsy model, along with upregulating expression of its targets $\mathrm{LXR} \alpha$ and SOCS1 in IBA1-labeled microglia in the hippocampus. Based on a key proinflammatory role of miR-155-5p in microglia, intranasal delivery of miR-155-5p antagomir likely improved acute seizures by blocking hippocampal inflammation. This study suggests that, in comparison with invasive delivery strategies, such as hippocampal microinjection, ${ }^{31}$ a more practical delivery strategy for the translational development of epilepsy therapies is possible.

Currently, glucocorticoids are widely used to attenuate inflammation in human diseases, but their chronic use is complicated by their side effects; ${ }^{3,32}$ thus, a series of other strategies have been employed to treat inflammatory injuries, such as overexpression of proteins that compete with proinflammatory cytokines to bind their receptors, such as IL-1 receptor antagonist protein, ${ }^{33}$ and decoy receptors that bind proinflammatory cytokines and chemokines but are unable to transduce signaling, thus acting as molecular traps to prevent proinflammatory ligands from interacting with their receptors. ${ }^{34}$ In addition, inflammation could be blocked by antiinflammatory proteins that inhibit cytokineinduced signal transduction and transcription, such as SOCS1 in the JAK/STAT pathway. ${ }^{35}$ However, these proteins are usually macromolecules, which are difficult to deliver and function in the brain because of their inability to permeate the BBB; thus, other methods should be developed to address these obstacles. Interestingly, miRNAs, a group of noncoding RNAs only 22 nucleotides 


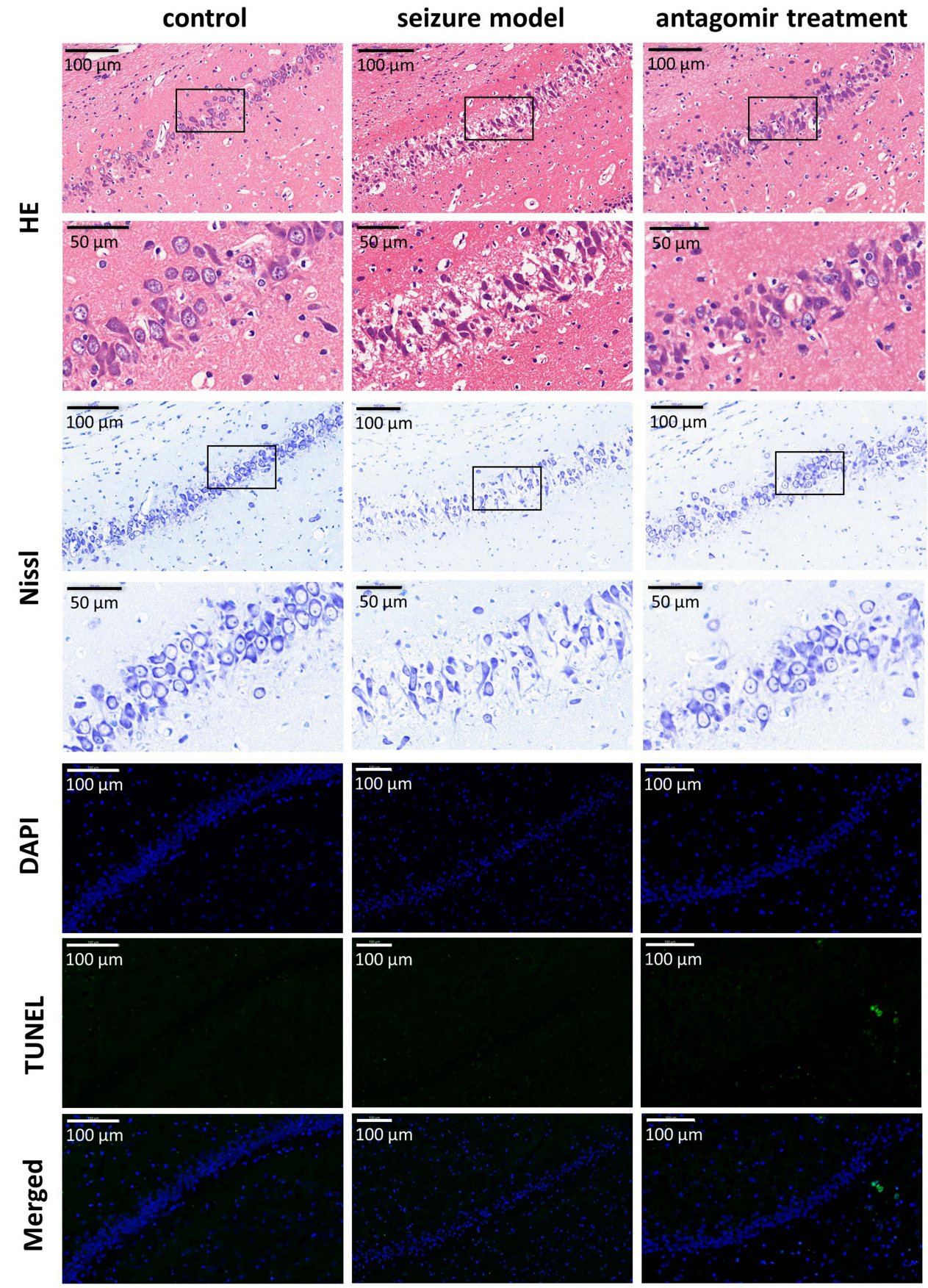

Figure 7 Protective effects of miR-155-5p antagomir in the hippocampus 60 min after pentetrazol administration. In comparison with the HE-stained hippocampal nuclei in the control group, the hippocampal nuclei (blue) in the seizure model group were much fainter, but the nuclei were relatively preserved by intranasal delivery of miR-I55-5p antagomir in the antagomir treatment group. Compared with the control group, the seizure model group exhibited significantly fewer Nissl bodies (blue) in hippocampal tissues, whereas this reduction was revered in the antagomir treatment group. No visible apoptosis (stained by TUNEL in blue) was observed in the hippocampal nuclei (stained by DAPI in green) of the control, seizure model or antagomir treatment groups.

in length, have been demonstrated to be able to bypass the BBB by intranasal delivery. ${ }^{18,19}$ Hence, miRNAs are considered potential targets in this study for modulating inflammation in epilepsy pathologies.

Several studies have recently reported that inhibition of miR-155-5p by its antagomir attenuates matrix metallopeptidase 3 overexpression after IL-1 $\beta$ stimulation in astrocytes, ${ }^{36}$ and the expression of miR-155-5p increased significantly in myeloid-related protein-8 induced astrocyterelated inflammation. ${ }^{37}$ In addition, miR-155-5p could be upregulated in amyloid $\beta$-activated astrocytes, thus contributing to the production of inflammatory mediators such as IL-6 
and interferon- $\beta{ }^{38}$ All the in vitro evidence indicates that astrocytic miR-155-5p participates in brain inflammation. However, its target mRNAs are still not determined in astrocytes. In comparison, microglia are well known to be the first line of the inflammatory response in the brain, and miR-155$5 \mathrm{p}$ has been demonstrated to facilitate inflammation in microglia via decreasing the expression of LXR $\alpha$, SOCS1 and receptor for activated $\mathrm{C}$ kinase 1 (RACK1). ${ }^{22,23,39}$ Intriguingly, RACK1 expression was upregulated in the brains of TLE patients, ${ }^{40}$ indicating that RACK1 is involved in other pathologies, as well as being negatively regulated by miR$155-5 \mathrm{p}$.

In our in vivo study, via identifying the expression levels of IL-1 $\beta$, TNF- $\alpha$ and IL- 6 in the hippocampus, we observed that miR-155-5p antagomir inhibited inflammatory response in the pentetrazol-induced epilepsy model. A series of experiments were then performed to determine whether the expression of LXR $\alpha$ and SOCS1 was upregulated by intranasal delivery of miR-155-5p antagomir. However, LXR $\alpha$ and SOCS1 expression levels in hippocampal tissues were altered with small magnitude by miR155-5p antagomir treatment in our study, partly because microglia are a small portion of the hippocampus, and any significant changes in microglia are difficult to detect in whole hippocampal tissues. Hence, double immunofluorescence labeling was further employed to observe the expression of LXR $\alpha$ and SOCS1 in IBA1-labeled microglial cells of the hippocampus. LXR $\alpha$ and SOCS1 expression in IBA1-labeled microglial cells of the hippocampus was higher in the epilepsy model treated with miR-155-5p antagomir than in the epilepsy model without treatment, which indicates that intranasal delivery of miR-155-5p antagomir alleviated seizures via inhibiting neuroinflammation mediated by LXR $\alpha$ and SOCS1. Notably, miR$155-5 p$ functions in biological processes by negatively modulating multiple target mRNAs. Therefore, where (in microglia, astrocytes and even neurons) and via which target molecules miR-155-5p functions in brain inflammation and epileptic pathologies needs to be investigated in the future.

In theory, miRNAs regulate biological activities by suppressing the translation of their target genes; thus, the function of miR-155-5p depends on its target genes. According to bioinformatic analysis, hundreds of genes have been predicted to be targets of miR-155-5p, and many of them have been confirmed in tumors, ${ }^{41,42}$ as well as inflammatory diseases, indicating that miR-155-5p is a multitarget modulator involved in various mechanisms. Cai et al previously observed that miR-155-5p antagomir could alleviate epileptic seizures by upregulating the expression level of brainderived neurotrophic factor (BDNF) in the TrkB signaling pathway, another target of miR-155-5 $\mathrm{p}^{31}$ indicating that intranasal delivery of miR-155-5p antagomir in the present study likely alleviated acute seizures not only through inflammatory modulation but also through other mechanisms. Hence, further research remains to be performed to clarify the mechanisms of miR-155-5p antagomir for epilepsy treatment. Due to the involvement of miR-155-5p in tumors, ${ }^{43}$ the long-term safety of miR-155-5p antagomir should also be further explored.

Certain limitations should be noted in the present study. First, we demonstrated that intranasal delivery of miR-155-5p antagomir improved epileptic seizures and hippocampal injury in the pentetrazol-induced model, supporting its value in alleviating acute convulsions, and therefore, future experiments should attempt to establish chronic spontaneous epilepsy model to achieve a stable miR-155-5p level. Moreover, a chronic spontaneous epilepsy model is more similar to human epilepsy. However, the epilepsy model requires an automatic 24-h monitoring system of animal behavior, and thus, we were unable to perform these experiments without such a system in the present study. Second, miR-155-5p agomir and negative control were not used in this study; miRNAs are a group of multi-target molecules, we incline to the view of no necessity to prove exclusive effects by extra comparison with scrambled miRNAs, because other miRNAs probably modulate the same target mRNAs of miR-155-5p, thus an agomir or an antagomir of other miRNAs could also result in similar effects observed in the present study by miR-155-5p antagomir. In addition, the level of miR-155-5p began to decrease significantly $30 \mathrm{~min}$ after intranasal delivery and maintained a similar level in the next $90 \mathrm{~min}$; thus, we observed the effect of miR-155-5p antagomir on epileptic seizures during this period in the study. However, any modifications of the protocol, such as changes in the examination time points after pentetrazol administration after intranasal delivery and the final volumes/dosages of miR-155-5p antagomir, may lead to incompletely consistent results.

\section{Conclusion}

This study first demonstrated that intranasal delivery of miR$155-5 \mathrm{p}$ antagomir alleviated acute seizures, likely by blocking hippocampal inflammation in the pentetrazol-induced 
model. Compared with invasive delivery such as hippocampal microinjection, intranasal delivery of miR-155-5p antagomir represents a more practical strategy for the translational development of epilepsy treatment. Nevertheless, other potential mechanisms of miR-155-5p antagomir and its longterm safety for epilepsy treatment remain to be explored.

\section{Acknowledgments}

This study was funded by the Postdoctoral Science Foundation of Guangdong Medical University (Grant No. 701z20150002), Medical Scientific Research Foundation of Guangdong Province of China (Grant No. A2019152), and the Natural Science Foundation of Guangdong Province (Grant No. 2017A030310658).

\section{Disclosure}

The authors declare no conflicts of interest in this work.

\section{References}

1. Perucca E, French J, Bialer M. Development of new antiepileptic drugs: challenges, incentives, and recent advances. Lancet Neurol. 2007;6:793-804. doi:10.1016/S1474-4422(07)70215-6

2. Vezzani A, French J, Bartfai T, Baram T. The role of inflammation in epilepsy. Nat Rev Neurol. 2011;7:31-40. doi:10.1038/nrneurol.2010.178

3. Najjar S, Bernbaum M, Lai G, Devinsky O. Immunology and epilepsy. Rev Neurol Dis. 2008;5:109-116.

4. Vincent A, Irani S, Lang B. The growing recognition of immunotherapy-responsive seizure disorders with autoantibodies to specific neuronal proteins. Curr Opin Neurol. 2010;23(2):144-150. doi:10.1097/WCO.0b013e32833735fe

5. Auvin S, Mazarati A, Shin D, Sankar R. Inflammation enhances epileptogenesis in the developing rat brain. Neurobiol Dis. 2010;40:303-310. doi:10.1016/j.nbd.2010.06.004

6. Walker L, Sills G. Inflammation and epilepsy: the foundations for a new therapeutic approach in epilepsy? Epilepsy Curr. 2012;12:8-12. doi:10.5698/1535-7511-12.1.8

7. Dubé C, Vezzani A, Behrens M, Bartfai T, Baram T. Interleukin1 beta contributes to the generation of experimental febrile seizures. Ann Neurol. 2005;57:152-155. doi:10.1002/ana.20358

8. Samland H, Huitron-Resendiz S, Masliah E, Criado J, Henriksen S, Campbell I. Profound increase in sensitivity to glutamatergic- but not cholinergic agonist-induced seizures in transgenic mice with astrocyte production of IL-6. J Neurosci Res. 2003;73:176-187. doi: $10.1002 /$ jnr. 10635

9. Akassoglou K, Probert L, Kontogeorgos G, Kollias G. Astrocytespecific but not neuron-specific transmembrane TNF triggers inflammation and degeneration in the central nervous system of transgenic mice. J Immunol. 1997;158(1):438-445.

10. Probert L, Akassoglou K, Pasparakis M, Kontogeorgos G, Kollias G. Spontaneous inflammatory demyelinating disease in transgenic mice showing central nervous system-specific expression of tumor necrosis factor alpha. Proc Natl Acad Sci U S A. 1995;92(24):11294-11298. doi:10.1073/pnas.92.24.11294

11. Wilcox KS, Vezzani A. Does brain inflammation mediate pathological outcomes in epilepsy? Adv Exp Med Biol. 2014;813:169-183.

12. Vezzani A, Balosso S, Ravizza T. The role of cytokines in the pathophysiology of epilepsy. Brain Behav Immun. 2008;22 (6):797-803. doi:10.1016/j.bbi.2008.03.009
13. De Simoni M, Perego C, Ravizza T, et al. Inflammatory cytokines and related genes are induced in the rat hippocampus by limbic status epilepticus. Eur J Neurosci. 2000;12(7):2623-2633. doi:10.1046/ j.1460-9568.2000.00140.x

14. Mazumder A, Sharma P, Patial V, Singh D. Ginkgo biloba L. attenuates spontaneous recurrent seizures and associated neurological conditions in lithium-pilocarpine rat model of temporal lobe epilepsy through inhibition of mammalian target of rapamycin pathway hyperactivation. $J$ Ethnopharmacol. 2017;204:8-17.

15. Riikonen R. Infantile spasms: therapy and outcome. J Child Neurol. 2004;19:401-404. doi:10.1177/088307380401900601

16. Wirrell E, Farrell K, Whiting S. The epileptic encephalopathies of infancy and childhood. Can J Neurol Sci. 2005;32:409-418. doi: $10.1017 / \mathrm{S} 0317167100004388$

17. Coondoo A, Phiske M, Verma S, Lahiri K. Side-effects of topical steroids: a long overdue revisit. Indian Dermatol Online J. 2014;5:416-425. doi:10.4103/2229-5178.142483

18. Hanson LR, Frey WH 2nd. Intranasal delivery bypasses the blood-brain barrier to target therapeutic agents to the central nervous system and treat neurodegenerative disease. BMC Neurosci. 2008;9 (Suppl 3):S5. doi:10.1186/1471-2202-9-S3-S5

19. Thorne RG, Pronk GJ, Padmanabhan V, Frey WH 2nd. Delivery of insulin-like growth factor-I to the rat brain and spinal cord along olfactory and trigeminal pathways following intranasal administration. Neuroscience. 2004;127:481-496. doi:10.1016/j.neuroscience.2004. 05.029

20. Lee ST, Jeon D, Chu K, et al. Inhibition of miR-203 reduces spontaneous recurrent seizures in mice. Mol Neurobiol. 2016.

21. Tao H, Zhao J, Liu T, et al. Intranasal delivery of miR-146a mimics delayed seizure onset in the lithium-pilocarpine mouse model. Mediators Inflamm. 2017;2017:6512620. doi:10.1155/2017/6512620

22. Cardoso AL, Guedes JR, Pereira de Almeida L, Pedroso de Lima MC. miR-155 modulates microglia-mediated immune response by down-regulating SOCS-1 and promoting cytokine and nitric oxide production. Immunology. 2012;135:73-88.

23. Li P, Wang G, Zhang X, et al. MicroRNA-155 promotes heat stressinduced inflammation via targeting liver $\mathrm{X}$ Receptor $\alpha$ in microglia. Front Cell Neurosci. 2019;13:12. doi:10.3389/fncel.2019.00012

24. Ashhab M, Omran A, Kong H, et al. Expressions of tumor necrosis factor alpha and microRNA-155 in immature rat model of status epilepticus and children with mesial temporal lobe epilepsy. $J \mathrm{Mol}$ Neurosci. 2013;51:950-958. doi:10.1007/s12031-013-0013-9

25. Thorne RG, Emory CR, Ala TA, Frey WH 2nd. Quantitative analysis of the olfactory pathway for drug delivery to the brain. Brain Res. 1995;692:278-282. doi:10.1016/0006-8993(95)00637-6

26. Caulder EH, Riegle MA, Godwin DW. Activation of group 2 metabotropic glutamate receptors reduces behavioral and electrographic correlates of pilocarpine induced status epilepticus. Epilepsy Res. 2014;108:171-181. doi:10.1016/j.eplepsyres.2013.10.009

27. Racine RJ. Modification of seizure activity by electrical stimulation. II. Motor seizure. Electroencephalogr Clin Neurophysiol. 1972;32:281-294. doi:10.1016/0013-4694(72)90177-0

28. Liu L, Hamre KM, Goldowitz D. Kainic acid-induced neuronal degeneration in hippocampal pyramidal neurons is driven by both intrinsic and extrinsic factors: analysis of $\mathrm{FVB} / \mathrm{N} \leftrightarrow \mathrm{C} 57 \mathrm{BL} / 6$ chimeras. $J$ Neurosci. 2012;32:12093-12101. doi:10.1523/JNEUROSCI.6478-11.2012

29. Djupesland PG, Messina JC, Mahmoud RA. The nasal approach to delivering treatment for brain diseases: an anatomic, physiologic, and delivery technology overview. Ther Deliv. 2014;5:709-733. doi:10.4155/tde.14.41

30. Lochhead JJ, Thorne RG. Intranasal delivery of biologics to the central nervous system. Adv Drug Deliv Rev. 2012;64:614-628. doi:10.1016/j.addr.2011.11.002

31. Cai Z, Li S, Li S, et al. Antagonist targeting microRNA-155 protects against lithium-pilocarpine-induced status epilepticus in C57BL/6 mice by activating brain-derived neurotrophic factor. Front Pharmacol. 2016;7:129. doi:10.3389/fphar.2016.00129 
32. Cruz-Topete D, Cidlowski JA. One hormone, two actions: anti- and pro-inflammatory effects of glucocorticoids. Neuroimmunomodulation. 2015;22:20-32. doi:10.1159/000362724

33. Dinarello C. Immunological and inflammatory functions of the interleukin-1 family. Annu Rev Immunol. 2009;27:519-550. doi:10. 1146/annurev.immunol.021908.132612

34. Mantovani A, Locati M, Vecchi A, Sozzani S, Allavena P. Decoy receptors: a strategy to regulate inflammatory cytokines and chemokines. Trends Immunol. 2001;22:328-336. doi:10.1016/S14714906(01)01941-X

35. Liau N, Laktyushin A, Lucet I, et al. The molecular basis of JAK/ STAT inhibition by SOCS1. Nat Commun. 2018;9(1):1558. doi:10.1038/s41467-018-04013-1

36. Korotkov A, Broekaart D, van Scheppingen J, et al. Increased expression of matrix metalloproteinase 3 can be attenuated by inhibition of microRNA-155 in cultured human astrocytes. J Neuroinflammation. 2018;15:211. doi:10.1186/s12974-018-1245-y

37. Kong H, Yin F, He F, et al. The effect of miR-132, miR-146a, and miR-155 on MRP8/TLR4-induced astrocyte-related inflammation. J Mol Neurosci. 2015;57:28-37. doi:10.1007/s12031-015-0574-x

38. Guedes J, Custódia C, Silva R, de Almeida L, Pedroso de Lima M, Cardoso A. Early miR-155 upregulation contributes to neuroinflammation in Alzheimer's disease triple transgenic mouse model. Hum Mol Genet. 2014;23:6286-6301.
39. Yin H, Song S, Pan X. Knockdown of miR-155 protects microglia against LPS-induced inflammatory injury via targeting RACK1: a novel research for intracranial infection. J Inflammation. 2017;14 (1):17. doi:10.1186/s12950-017-0162-7

40. Chen Y, Huang XJ, Yu N, et al. HMGB1 contributes to the expression of P-glycoprotein in mouse epileptic brain through toll-like receptor 4 and receptor for advanced glycation end products. PLoS One. 2015;10.

41. Ji Y, Fioravanti J, Zhu W, et al. miR-155 harnesses Phf19 to potentiate cancer immunotherapy through epigenetic reprogramming of CD8 T cell fate. Nat Commun. 2019;10:2157. doi:10.1038/s41467019-09882-8

42. Wu Q, Sun S, Li Z, et al. Tumour-originated exosomal miR-155 triggers cancer-associated cachexia to promote tumour progression. Mol Cancer. 2018;17:155. doi:10.1186/s12943-018-0899-5

43. Jayawardana K, Schramm S, Tembe V, et al. Identification, review, and systematic cross-validation of microRNA prognostic signatures in metastatic melanoma. J Invest Dermatol. 2016;136(1):245-254. doi:10.1038/JID.2015.355
Neuropsychiatric Disease and Treatment

\section{Publish your work in this journal}

Neuropsychiatric Disease and Treatment is an international, peerreviewed journal of clinical therapeutics and pharmacology focusing on concise rapid reporting of clinical or pre-clinical studies on a range of neuropsychiatric and neurological disorders. This journal is indexed on PubMed Central, the 'PsycINFO' database and CAS, and
Dovepress

is the official journal of The International Neuropsychiatric Association (INA). The manuscript management system is completely online and includes a very quick and fair peer-review system, which is all easy to use. Visit http://www.dovepress.com/testimonials.php to read real quotes from published authors. 